\title{
Spectroscopy and photodissociation of chlorine monomers and clusters in argon matrices
}

\author{
J. G. McCaffrey, ${ }^{a)}$ H. Kunz, and N. Schwentner \\ Institut für Experimentalphysik, Freie Universität Berlin, Arnimallee 14, D-1000 Berlin 33, Germany
}

(Received 24 June 1991; accepted 16 September 1991)

\begin{abstract}
Steady-state and time-resolved emission spectroscopy of the $A-X$ system of $\mathrm{Cl}_{2}$ is used to distinguish molecular chlorine isolated as monomers and clusters in solid argon samples. The lifetime of the metastable $A^{\prime}{ }^{3} \Pi_{2 u}$ state is measured to be an order of magnitude less, when chlorine is present as clusters, than when it is truly isolated. Photodissociation of molecular chlorine clusters was found to be insignificant as monitored by the emission of $\mathrm{Ar}_{2} \mathrm{Cl}$ at 260 $\mathrm{nm}$. Measurement of the dissociation threshold of molecular chlorine in the $9 \mathrm{eV}$ region as a function of temperature showed little variation. Using spectroscopic data it is concluded that dissociation is occurring by an impulsive mechanism involving curve-crossing from the initially populated $\mathrm{Ar}^{+}\left(\mathrm{Cl}_{2}\right)^{-}$charge transfer state to repulsive potentials correlating with ground state atomic chlorine and not via a harpooning mechanism. A simple microscopic model, drawn from experimental data and pairwise addition of $\mathrm{ArCl}$ potential terms, is constructed to describe the steps involved in this dissociation process in the solid lattice.
\end{abstract}

\section{INTRODUCTION}

As a model for the relaxation dynamics of small molecules in condensed matter, matrix-isolated chlorine has been the subject of considerable interest. Work in this area includes spectroscopic investigations of Bondybey et al. ${ }^{1}$ and later by the Schurath group ${ }^{2}$ on the $B$ and $X$ states of molecular chlorine as well as examinations of the "cage effect" by Flynn's ${ }^{3}$ and Apkarian's ${ }^{4}$ groups. More recently molecular dynamics calculations by Gerber and co-workers ${ }^{5}$ have directly addressed the phenomenon of the matrix "cage effect" on molecular dissociation. In a previous paper ${ }^{6}$ on the photodissociation of $\mathrm{Cl}_{2}$ in argon matrices, we have described the dependence of fragmentation efficiency on sample crystallinity and photon energy. In this paper we present in more detail the dependence of molecular chlorine dissociation on excitation wavelength as well as a function of temperature. We also examine cluster formation of molecular chlorine in solid argon and its possible involvement in the photochemistry. Following these studies we are confident that the photochemistry being described in our experiment pertains to that of truly isolated molecular chlorine, thus allowing a meaningful comparison with theory regarding the dissociation mechanism.

The structure of this paper is as follows: the experimental section contains details of the additional equipment used principally to detect the presence of molecular chlorine clusters. Description of these cluster measurements is contained in the first section of the Results followed by details of the dissociation curve and its temperature dependence. In the Discussion section we present a simple microscopic model of the dissociation and justify, using arguments obtained from spectroscopic data, our conclusion that the dissociation observed in Ar occurs by a curve crossing mechanism. The

"Alexander von Humboldt Fellow 1989-1991. Permanent address; Department of Chemistry, St. Patrick's College, Maynooth, Co. Kildare, Ireland.
Conclusion brings together results obtained in our earlier paper on this subject as well as the more detailed results of the present study.

\section{EXPERIMENT}

As stated in the preceeding article ${ }^{6}$ of this series, the identification of molecular chlorine photochemistry is facilitated by monitoring the intensity of the atomic chlorine related $\mathrm{Ar}_{2} \mathrm{Cl}$ emission at $260 \mathrm{~nm}$, induced by excitation at 168 $\mathrm{nm}$. Molecular chlorine can be detected in one of two ways; first by monitoring the $A^{\prime}{ }^{3} \Pi_{2 u}-X^{1} \Sigma_{0 g}^{+}$emission (henceforth abbreviated as the $A-X$ emission) in the red/near-infrared region or second by the $\mathrm{ArCl}_{2}$ emission at $360 \mathrm{~nm}^{7}$ Photochemical studies were made using the $3 \mathrm{mNIM}-\mathrm{II}$ monochromator at the synchrotron source BESSY in Berlın and unless otherwise stated, laser excitation at $308 \mathrm{~nm}$ was used exclusively for spectroscopic purposes, i.e., in producing $A-X$ emission of $\mathrm{Cl}_{2}$.

Details of the experimental apparatus used in this study have been presented in our earlier paper on $\mathrm{Cl}_{2}$ dissociation in argon matrices ${ }^{6}$ and only the additional equipment used in this study will be described here. A B\&M Spektronik OSA 500 optical spectrum analyzer, having a 512 diode array unit, was used to detect the $A-X$ emission of $\mathrm{Cl}_{2}$ produced by excimer laser excitation at $308 \mathrm{~nm}$. Spectral calibration of the diode array camera was made using the output of a Lott krypton pen lamp. The geometry used in this configuration involved the use of a $0.25 \mathrm{~m}$ Ebert monochromator orthogonal to the laser axis. The two optical axes were coupled by a 1:1 lens focusing the emitted light onto the entrance slit of the monochromator whose exit slit was replaced by the diode array camera. The $\mathrm{XeCl}$ excimer laser used was a Lambda Physik EMG 53MSC the output of which was loosely focused with a long focal length lens onto the sample. Spectral resolution in emission is considerably better using this configuration than that achievable in the synchrotron measurements for the reason that an entrance slit is used in the 
former setup. Use of an entrance slit is avoided in the synchrotron experiment to improve the signal-to-noise ratio and as a result, the spectral resolution in emission is limited by the spatial extension of the focus of the synchrotron light.

Growth of bulk crystals involved the condensation of the rare gas/chlorine mixtures in a quartz holder and the subsequent removal of the holder leaving a free standing crystal. ${ }^{8}$ The deposition temperature was typically $15 \mathrm{~K}$ but the temperature at the condensation front is known to be considerably higher. ${ }^{8}$ All the thin film samples were grown by slow deposition $(5 \mathrm{mmol} / \mathrm{h})$ at $18 \mathrm{~K}$ and had thicknesses ranging from 10 to $50 \mu \mathrm{m}$ depending on the $\mathrm{Cl}_{2}$ concentration used.

\section{RESULTS}

\section{A. Spectroscopic identification of monomers and clusters}

The behavior of matrix-isolated molecular chlorine with respect to cluster formation was examined in two different experiments. One involved the deposition of $\mathrm{Cl}_{2} / \mathrm{Ar}$ mixtures as thin films the other yielding free standing crystals. Due to the very different sample preparation conditions, i.e., flow rates and deposition temperatures, it is expected that samples having very different morphology will result. In trace (a) of Fig. 1, we show the $A-X$ emission system recorded using an array camera and excited by 308 nm laser excitation for a free standing crystal with a concentration of $0.01 \% \mathrm{Cl}_{2} / \mathrm{Ar}$. Trace (c) shows the same emission system for a sample of similar concentration but this time grown as a thin film. The latter spectrum exhibits the isotope-related splitting and relative intensities expected for the $A-X$ emission band of $\mathrm{Cl}_{2}$. As the chlorine concentration is further reduced the isotopic splitting becomes more pro-

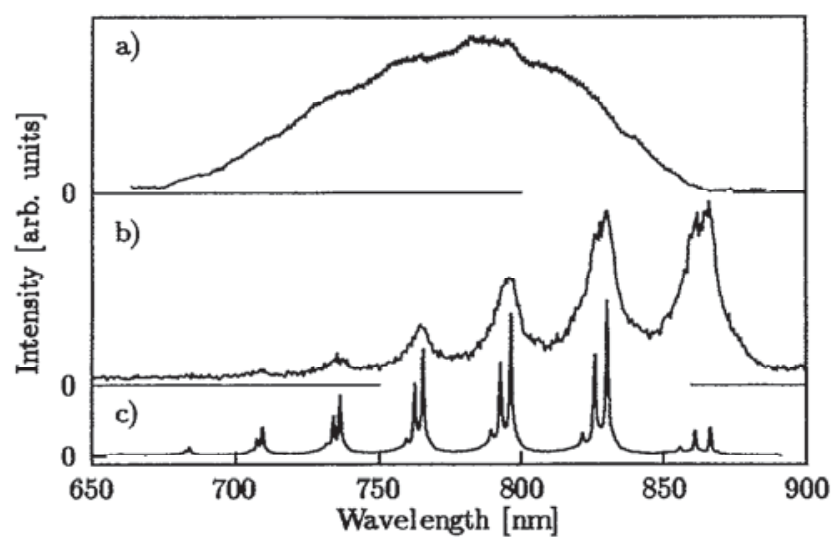

FIG. 1. The contributions of chlorine clusters in the $A-X$ emission of $0.01 \%$ $\mathrm{Cl}_{2} / \mathrm{Ar}$ samples under different deposition and detection conditions. Trace (a) is the laser-induced emission of a free standing crystal detected by a diode array camera. Traces (b) and (c) are thin film samples recorded with synchrotron/photomultiplier and laser/camera detection systems, respectively. The different intensity distribution in the spectrum shown in trace (b) arises from the different wavelength response functions of the RCA C31034-02 photomultiplier used in comparison to the B\&M Spektronik 500 optical spectrum analyzer used to detect traces (a) and (b). nounced. This observation is in agreement with the observations of Andrews and co-workers ${ }^{9}$ in the Raman and fluorescence spectroscopy of matrix-isolated molecular chlorine. Based on this concentration dependence and from a comparison with data published by Apkarian and coworkers ${ }^{10}$ on the emission of $\mathrm{Cl}_{2}$ in solid chlorine, it can be concluded that concentrations of $0.01 \%$ in thin films, yield chlorine molecules isolated predominantly as monomers. Trace (b) of Fig. 1 shows a comparable emission profile recorded using synchrotron radiation and presented in our previous paper on molecular chlorine dissociation. ${ }^{6}$ The lack of well resolved isotopic structure in this spectrum is, as explained in the experimental section, a result of the reduced spectral resolution inherent in the optical configuration used and not related to the formation of significant amounts of clusters.

In order to identify contributions from monomer and cluster emission in the $A-X$ system we have monitored this emission for specific delay times using the inherent time resolution of the array camera to create time windows of $32 \mathrm{~ms}$. This technique exploits the forbidden nature of the $A^{\prime 3} \mathrm{I}_{2 u}-X^{1} \Sigma_{g}^{+}$emission of $\mathrm{Cl}_{2} \cdot{ }^{1}$ The metastable $A^{\prime}{ }^{3} \Pi_{2 u}$ state is accessed indirectly by laser excitation to the repulsive $C^{1} \Pi_{1 u}$ state whose population subsequent to recombination and matrix induced relaxation, ends up in the lowest excited $A^{\prime}$ state. ${ }^{1}$ The sensitivity of the long $A^{\prime}{ }^{3} \Pi_{2 u}$ state radiative lifetime [ $76 \mathrm{~ms}$ (Ref. 1) ] to its environment, allows the form in which $\mathrm{Cl}_{2}$ is isolated in the solid to be identified. Traces (a) and (b) in Fig. 2 show the emission profiles recorded for the $A-X$ system for progressively longer delay times. In trace (a) the prompt emission spectrum is shown while (b) shows that recorded after a delay of $200 \mathrm{~ms}$. It can be clearly seen that at short time, a structureless profile dominates while at longer times, the structured profile of the isolated $\mathrm{Cl}_{2}$ (see Fig. 1) is seen exclusively. This behavior reflects the fact that the forbiddeness of the transition is diminished, thereby shortening the radiative lifetime, when molecular chlorine is present as clusters.

Another manifestation of the presence of clusters in ar-

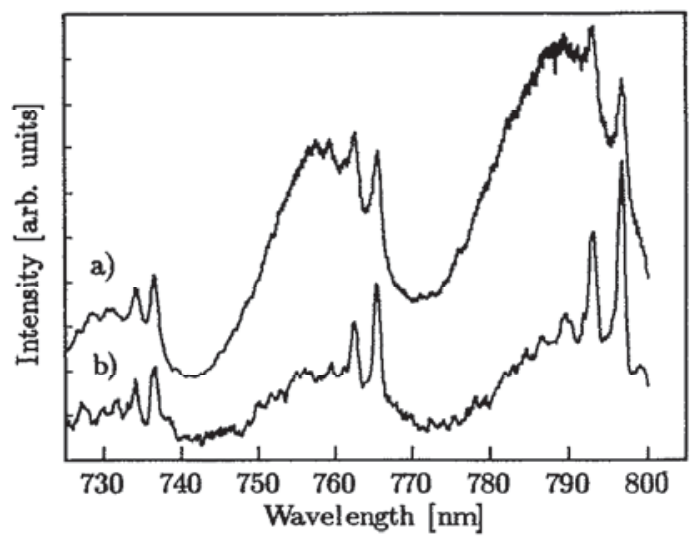

FIG. 2. Time-resolved emission spectra of the $A-X$ sxstem of $\mathrm{Cl}_{2} / \mathrm{Ar}$ recorded for (a) zero and (b) $200 \mathrm{~ms}$ delay times of the array camera. Note the dominance of the broad featureless bands at early times and the increasing intensity of the sharp monomer related species at longer times. 
gon samples containing more than $0.1 \% \mathrm{Cl}_{2}$ can be seen by monitoring the $A-X$ emission as a function of different excitation wavelengths. Figure 3 shows the form of the $A-X$ emission following excitation at (a) 130, (b) 330, and (c) $180 \mathrm{~nm}$. The most isotopically resolved emission profiles are recorded for excitation at 130 or $330 \mathrm{~nm}$ while the structureless and blue-shifted emission is most pronounced for excitation at $180 \mathrm{~nm}$. The isotopically resolved bands are assigned as emission originating from truly isolated $\mathrm{Cl}_{2}$ molecules while the blue-shifted emission originates from a mixture of cluster and monomer emission. Excitation spectra of the different emitting species (indicated by the arrows in Fig. 3 ) in the $A-X$ emission are presented in Fig. 4. From Fig. 4 it is clear that the $180 \mathrm{~nm}$ band contributes predominantly to structureless emission in contrast to the $130 \mathrm{~nm}$ band. Although not shown in Fig. 4, the behavior of excitation at 330 $\mathrm{nm}$ is identical to that of the $130 \mathrm{~nm}$ band.

\section{B. Photochemistry of clusters}

The photochemistry of molecular chlorine clusters in Ar samples containing $0.5 \% \mathrm{Cl}_{2}$ was examined by checking for the production of atomic chlorine following prolonged irradiation with undispersed synchrotron light (i.e., zero order of the 3 mNIM II monochromator) using optical filters to select wavelength ranges. The wavelength regions examined in this way are in order of increasing energy; the visible UV $(\lambda>280 \mathrm{~nm})$, selected with a $280 \mathrm{~nm}$ cutoff filter (WG $280)$; the UV region $(\lambda>155 \mathrm{~nm})$, selected with quartz and the vacuum UV region ( $\lambda>105.2 \mathrm{~nm}$ ), selected using LiF. The results of photolysis of these cluster-containing samples for all of these spectral regions were similar in that the production of atomic chlorine, as monitored by the $\mathrm{Ar}_{2} \mathrm{Cl}$ emission at $260 \mathrm{~nm}$, was miniscule.

The difference in the photochemical behavior between molecular chlorine isolated as monomer and in clusters is manifested by the selective bleaching of monomeric $\mathrm{Cl}_{2}$ following irradiation. Shown in Fig. 5 are excitation spectra recorded for the cluster specific $\mathrm{Cl}_{2} A-X$ emission before and after irradiation with undispersed light of a sample containing a significant amount of molecular chlorine clusters. Thus

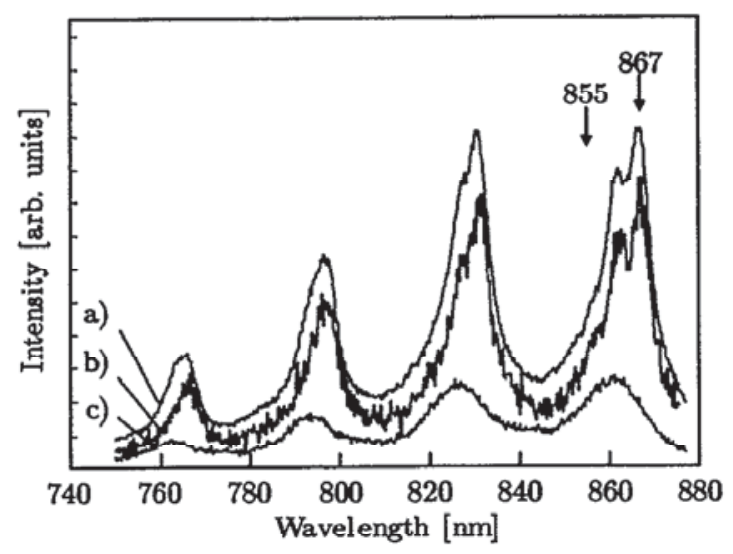

FIG. 3. Emission spectra of the $\mathrm{Cl}_{2} A-X$ system recorded with synchrotron radiation following excitation at $130 \mathrm{~nm}$ trace (a), $330 \mathrm{~nm}$ trace (b), and $180 \mathrm{~nm}$ trace (c).

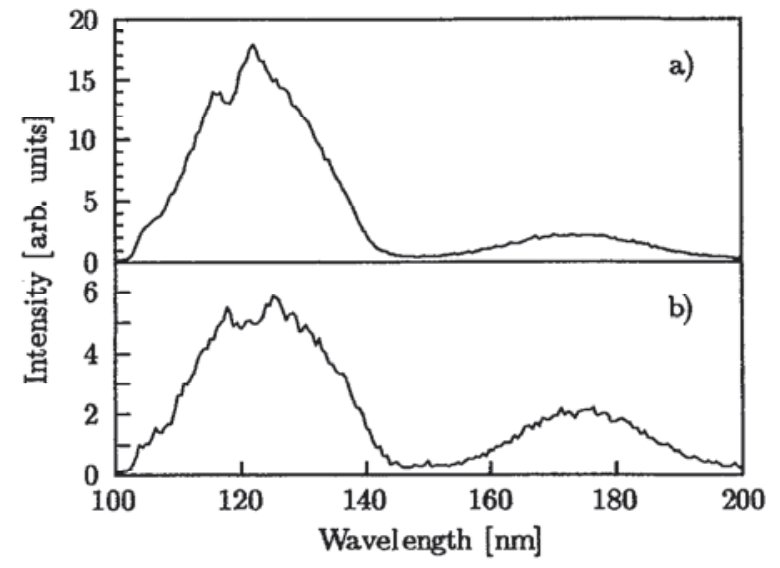

FIG. 4. Excitation spectra of the dominant features in the $v^{\prime}=0 \rightarrow v^{\prime \prime}=8$ vibrational band shown in Fig. 3 . Trace (a) corresponds to the $A-X$ emission originating from monomers $\left(\lambda_{\mathrm{em}}=867 \mathrm{~nm}\right)$. Trace $(\mathrm{b})$ is that related to cluster emission $\left(\lambda_{\mathrm{em}}=855 \mathrm{~nm}\right)$.

it can be seen in Fig. 5 that the intensity of the predominantly monomeric absorption at $130 \mathrm{~nm}$, monitored at $867 \mathrm{~nm}$ and shown by traces (a) and (b), is considerably depleted after irradiation. This in comparison with the $180 \mathrm{~nm}$ band, monitored at $855 \mathrm{~nm}$ and shown by traces (c) and (d), which originates from absorption of monomers and clusters where little change is seen. An attempt was made to identify this selective bleaching process in the emission of the $A-X$ system but was not successful duc to the limited spectral resolution obtained in emission spectra which is inherent in the synchrotron setup.

\section{C. $\mathrm{Cl}_{2}$ monomers photochemistry \\ 1. Crystalline samples}

The measurement of dissociation thresholds reported in our previous paper ${ }^{6}$ has been repeated in this study and ex-



FIG. 5. Photobleaching observed in the excitation spectra shown in Fig. 4 before, traces (a), (c), and after, traces (b), (d), irradiation with undispersed light. Note the depletion in the monomer excitation feature centered at $122.5 \mathrm{~nm}$ (recorded for $\lambda_{\mathrm{em}}=867 \mathrm{~nm}$ ) shown in the upper panel and the unchanged nature of the cluster emission (recorded for $\lambda_{\mathrm{em}}=855 \mathrm{~nm}$ ) shown in the lower panel after identical irradiation conditions. 
tended to check for temperature dependence and to examine the form of the threshold curves at different temperatures. It is however instructive to first compare an excitation spectrum of the $\mathrm{ArCl}_{2}$ emission at $360 \mathrm{~nm}$ with the dissociation efficiency curve of $\mathrm{Cl}_{2}$ in the region of $9 \mathrm{eV}$. Figure 6 shows such a comparison and it is immediately obvious that a large dip in the $\mathrm{ArCl}_{2}$ excitation spectrum (broken line) corresponds to the region of most efficient $\mathrm{Cl}_{2}$ dissociation in the threshold curve (points). Shown also in Fig. 6 (solid line) is the excitation spectrum of the $A-X$ system of $\mathrm{Cl}_{2}$ monitored by the $v^{\prime}=0 \rightarrow v^{\prime \prime}=8$ transition at $867 \mathrm{~nm}$. It can clearly be seen in Fig. 6 that the $A-X$ excitation profile exhibits antagonistic behavior with respect to the $\mathrm{ArCl}_{2}$ excitation profile. In other words, the $A-X$ system shows an intensity maximum at $10 \mathrm{eV}$ where the $\mathrm{ArCl}_{2}$ system shows an intensity dip. The correlation between these processes can be rationalized when the large quantum yield $(0.3)$ measured for the dissociation of $\mathrm{Cl}_{2}$ in this spectral region is considered. Thus, competitive branching between the dark dissociation channel and the radiative channel yielding $\mathrm{ArCl}_{2}$ emission at $360 \mathrm{~nm}$ leads to an intensity dip in the latter. Conversely, the presence of an additional channel feeding the $A$ state of $\mathrm{Cl}_{2}$, derived from the recombination of incompletely dissociated atomic chlorine fragments, leads to an enhancement of the $A-X$ emission intensity in the region of efficient dissociation. The correlation between these processes will be discussed in more detail ahead in relation to the dissociation mechanism.

The shape of the dissociation efficiency curve was examined in more detail with the aim of fitting this curve to simple analytical expressions describing dissociation. ${ }^{11}$ Such a curve is shown in Fig. 7 for two extreme temperature values, i.e., 5 and $23 \mathrm{~K}$. For both curves the small residual dissociation related to the $180 \mathrm{~nm}$ band has been subtracted so that the true behavior of the $130 \mathrm{~nm}$ band can be examined. At-

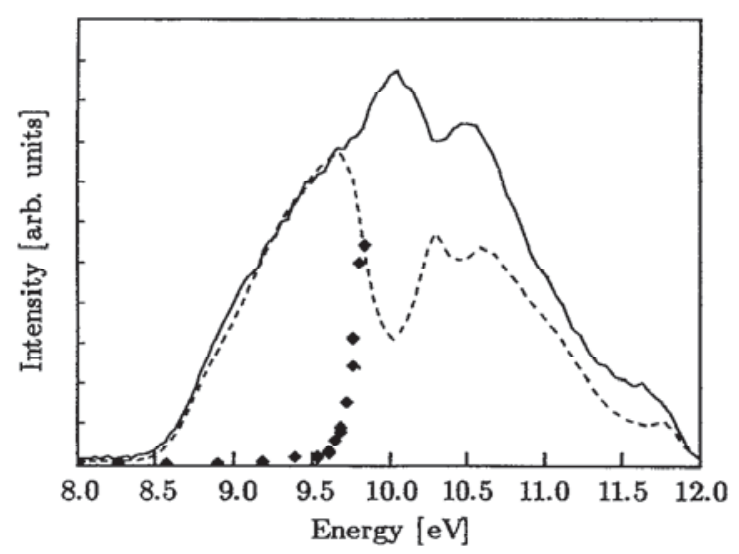

FIG. 6. Relationship between the dominant decay channels of molecular chlorine following absorption into the bound $1 \Sigma_{\mu}^{+}$state. The dashed line represents the radiative decay channel recorded as the excitation spectrum of the $\mathrm{ArCl}_{2}$ emission at $360 \mathrm{~nm}$. The solid line is the excitation spectrum of the $A-X$ system of $\mathrm{Cl}_{2}$, monitored for the $v^{\prime}=0 \rightarrow v^{\prime \prime}=8$ transition at 867 $\mathrm{nm}$. The points are the dissociation efficiency as a function of photon excitation energy. All excitation features have been corrected for photon flux and scattering.

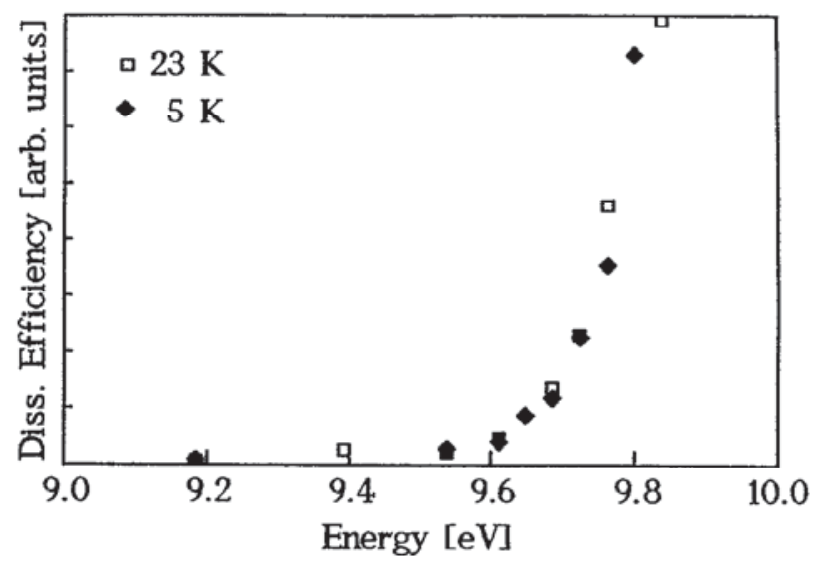

FIG. 7. The dissociation efficiency curve of $\mathrm{Cl}_{2}$ in argon recorded with high energy resolution and as a function of temperature. All points have been averaged over at least two consecutive measurements in the range of linear response to the photolysis flux.

tempts to fit the dissociation to the model applied to the $\mathrm{H}_{2} \mathrm{O} / \mathrm{RG}$ systems ${ }^{11}$ were however unsuccessful. It should be noted that the dissociation efficiency in this case has been evaluated for chlorine atoms occupying thermally stable sites, i.e., by monitoring the emission intensity after annealing to $28 \mathrm{~K}$. Furthermore, rigorous checks were made during measurement to obtain meaningful error limits and to ascertain that photochemistry in the range linear in photon flux was being made. As made evident in Fig. 7, there appears to be no variation in the threshold values or efficiencies within the limited temperature range examined.

\section{Noncrystalline samples}

The spectroscopy of atomic chlorine produced by photodissociation in noncrystalline samples (i.e., thin films deposited quickly $50 \mathrm{mmol} / \mathrm{h}$ and at $5 \mathrm{~K}$ ) has provided clues to the details of the dissociation mechanism and will be briefly described here. In panel (a) of Fig. 8, the excitation spectrum recorded after photolysis for $\mathrm{Ar}_{2} \mathrm{Cl}$ emission at $260 \mathrm{~nm}$ is presented. The features at lower energy are the $D-X$ and $B-X$ transitions of $\mathrm{ArCl}$ while the multiple line features at higher energies are ${ }^{2} P-{ }^{2} P$ resonance transitions from chlorine atoms ${ }^{12}$ occupying different sites. Annealing of such a sample produces the spectrum shown in panel (b) where the number of lines in the $\mathrm{Cl}^{2} P{ }^{2} P$ region has been reduced to a dominant line with a weak sideband, while the splitting between the $D-X$ and $B-X$ systems of $\mathrm{ArCl}$ is more pronounced. For comparison purposes the excitation spectrum recorded for the $260 \mathrm{~nm}$ emission in crystalline argon samples is shown in panel (c) and is clearly identical to that recorded for an annealed sample prepared under noncrystalline deposition conditions.

\section{DISCUSSION}

\section{A. Spectroscopy and photochemistry of clusters}

An interesting and yet somewhat puzzling result of our study of the chlorine cluster formation in argon matrices is the extent to which it occurs, particularly so in bulk crystals. 




FIG. 8. Excitation spectra of the $\mathrm{Ar}_{2} \mathrm{Cl}$ emission at $260 \mathrm{~nm}$ for (a) noncrystalline samples and (b) annealed noncrystalline samples after $\mathrm{Cl}$ atom production. Trace (c) is a crystalline sample directly after irradiation and without annealing.

This behavior is rather surprising in so far as the $\mathrm{Cl}_{2}$ molecule, with no permanent dipole moment, would be expected to have only weak intermolecular attraction, i.e., van der Waals bonding, originating principally from dispersive forces. One would as a result expect a statistical distribution of molecular chlorine in the growing crystal or thin film. Clearly this is not at all the case as shown in Fig. 1(a) for a 1:10000 dilution ratio gas mixture grown as a bulk crystal. As shown in trace (c) of Fig. 1, the extent of cluster formation can be minimized and practically eliminated in the case of samples grown as thin films by choosing the appropriate concentrations, deposition rates and temperatures.

Another rather puzzling behavior of molecular chlorine isolated as clusters is that it appears to exhibit remarkably diminished photochemical activity. The spectroscopy of $\mathrm{Cl}_{2}$ clusters in the $A-X$ system would indicate that the intermolecular interaction between the chlorine molecules constituting a cluster is strong enough to wipe out the isotopic structure (Fig. 1) and to shorten the $A^{\prime 3} \Pi_{2 u}$ state lifetime by an order of magnitude (Fig. 2). Evidence for similar increases in the oscillator strength of forbidden transitions of molecular chlorine, when isolated as clusters, are to be seen in Fig. 4 where the increased intensity of the ${ }^{3} \Sigma_{u}^{+} \leftarrow X^{1} \Sigma_{g}^{+}$system centered at $180 \mathrm{~nm}$ is obvious. This interaction between the constituent chlorine molecules in a cluster should also result in more efficient energy relaxation occuring either inter- or intramolecularly.

Other possible explanations for diminished photochemical activity might involve hindered cage exit or enhanced cage-induced recombination following dissociation. However, a very important point which must not be lost sight of, when discussing the photochemical activity of molecular chlorine clusters, is that for the detection of permanent $\mathrm{Cl}_{2}$ dissociation, it must be possible to build the $\mathrm{Ar}_{2} \mathrm{Cl}$ species and then emit as such. In other words dissociation occuring in a cluster environment may remain undetected since the $\mathrm{Ar}_{2} \mathrm{Cl}$ species cannot be formed. Such undetected chlorine atoms provide a possible explanation for the myriad of thermoluminecsence and long lived, diffusion rate limited emissions frequently observed directly after irradiation of rare gas solids containing halogens.

\section{B. Dissociation model}

As presented above, the photodissociation of $\mathrm{Cl}_{2}$ in crystalline argon samples produces features in the region of the atomic $\mathrm{Cl}^{2} P_{-}{ }^{2} P$ resonance, ${ }^{12}$ shown by trace (c) in Fig. 8 , identical to those in annealed noncrystalline samples, trace (b). To explain this behavior it is necessary to consider the packing of atomic and molecular chlorine in crystalline argon samples. The equilibrium internuclear separation of the $\mathrm{ArCl}$ van der Waals molecule in the $X^{2} \Sigma_{1 / 2}$ state (labeled $V_{3 / 2,1 / 2}$ in Appendix $\mathrm{A}$ and shown in Fig. 9 as the $|3 / 2,1 / 2\rangle$ state) is $3.78 \AA{ }^{13}$ Given that the substitutional site diameter in solid argon is $3.75 \AA .,{ }^{14}$ it is likely that the site giving rise to the dominant feature in the annealed sample (see Fig. 8) is a chlorine atom isolated in a single substitutional site. Furthermore, from the spectroscopic data of Janda and co-workers on the $\mathrm{ArCl}_{2}$ van der Waals molecule, ${ }^{15}$ the minimum $\mathrm{Ar}$ to $\mathrm{Cl}_{2}$ center-of-mass distance, in this " $T$ " shaped molecule, is $4.02 \AA$. From these size considerations, the isolation of $\mathrm{Cl}_{2}$ in $\mathrm{Ar}$ on deposition, must be in a divacancy site to avoid disruption of the lattice structure. Regarding the photodissociation process, the possibility exists therefore for the isolation of the two chlorine atoms in equivalent substitutional sites from the molecular parent which occupied a divacancy site.

These deductions are corroborated by our recent spectroscopic studies of $\mathrm{Cl}$ in the $\mathrm{HCl} / \mathrm{Ar}$ system following the dissociation of $\mathrm{HCl} .{ }^{16} \mathrm{Cl}$ atom spectroscopy in the $\mathrm{HCl} / \mathrm{Ar}$ system was found to be identical to that of the $\mathrm{Cl}_{2} / \mathrm{Ar}$ system but in the former system, the lattice occupancy is more clear cut than for $\mathrm{Cl}_{2}$ in $\mathrm{Ar}$. Thus $\mathrm{HCl}$ fits into a substitutional site

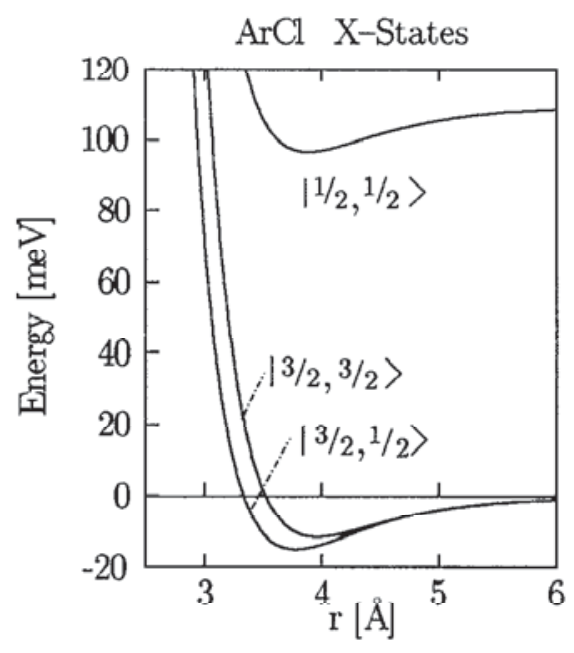

FIG. 9. Ground state potentials of $\mathrm{ArCl}$ originating from the interaction of $\mathrm{Cl}^{2} P_{1 / 2}$ and ${ }^{2} P_{3 / 2}$ with ground state argon. The curves, labeled $\left|j m_{j}\right\rangle$ for the situation of "weak field" coupling of the ${ }^{2} P_{j}$ atomic chlorine state, have been generated using the formalism of Acquilanti and co-workers, Refs. 13 and 17, and the data given in Appendix A. 
of argon and its dissociation products also fit into well defined lattice sites, viz., $\mathrm{H}$ into an octahedral interstitial site and $\mathrm{Cl}$ remaining in the original site of $\mathrm{HCl}$ isolation.

By considering the similarity in masses of atomic chlorine and argon it can be stated that head-on collisions result in very efficient transfer of kinetic energy between the two species. With regard to the escape of a chlorine atom in the dissociation of $\mathrm{Cl}_{2}$ in the matrix, this situation dictates that considerable kinetic energy is required and very few collisions can be tolerated in achieving successful cage escape. Molecular dynamics calculations of $\mathrm{Cl}_{2}$ dissociation in xenon, ${ }^{5}$ where the energy transfer efficiency is less than in argon, verify this prediction as they show a maximum of only two collisions with the host atoms in the dissociative trajectories. With such restrictions on the dissociation, it is likely that the orientation of the $\mathrm{Cl}_{2}$ molecule in the divacancy site of isolation plays a critical role in the photochemistry.

Using the experimental threshold data, the foregoing interpretation of the site occupancy and a reliable estimate of the $\mathrm{ArCl}$ pair potential, a reconstruction of the first steps taking place in the process of $\mathrm{Cl}_{2}$ dissociation in solid argon can be proposed. The potential used for $\mathrm{ArCl}$ is a modified version of that obtained from beam scattering data by Aquilanti and co-workers. ${ }^{13,17}$ This potential, as described in Appendix A, is modified from the Morse-spline-van der Waals potential of Aquilanti ${ }^{17}$ in that an exponential term has been included in an attempt to describe the repulsive interaction at short internuclear separation (which is of interest in this study) more accurately. Plots of the three potentials arising from the interaction of $\mathrm{Cl}^{2} P_{3 / 2}$ and $\mathrm{Cl}^{2} P_{1 / 2}$ with $\mathrm{Ar}^{1} S_{0}$ are shown in Fig. 9.

From the lattice parameters of solid argon, values of the $\mathrm{ArCl}$ internuclear separation incurred in the migration of atomic chlorine from the original site of isolation to its final site can be deduced. Choosing the minimum value of the $\mathrm{ArCl}$ separation for several possible pathways, we have used the pair potentials for the $X^{2} \Sigma_{1 / 2}$ state (labeled the $|3 / 2,1 / 2\rangle$ state of $\mathrm{ArCl}$ in Fig. 9) and summed over terms appropriate for the given pathway to estimate the energetic barrier experienced in such a migration. The geometry found which yields a barrier comparable to the experimentally observed threshold, is the movement of a chlorine atom through a triangular window and temporarily occupying an interstitial site. The calculated barrier is $7.7 \mathrm{eV}$ compared with the experimentally observed value of $6.8 \mathrm{eV}$, which is the observed threshold value $(9.3 \mathrm{eV})$ minus the binding energy of molecular chlorine $(2.5 \mathrm{eV})$. This mechanism involves initially the occupation of $\mathrm{Cl}_{2}$ in a divacancy site and finally the two chlorine atoms occupy substitutional sites separated by an argon atom.

Clearly the model used is very simple and has the serious shortcoming that it does not account for the additional threshold at $6.5 \mathrm{eV}$ with a small dissociation efficiency. It should also be emphasized that this is a static model in which the equilibrium positions of the argon atoms in the lattice are kept fixed. This consideration provides an obvious explanation of the fact that the calculated threshold is considerably greater than the experimentally observed value, viz., the geometry of the argon lattice has not been allowed to relax during atomic chlorine migration through the spatially restrictive triangular window. It is envisioned that molecular dynamics calculations on this system will provide a more realistic view of the intimate steps in the dissociation process.

\section{Dissociation mechanism}

An important aspect of molecular chlorine fragmentation at $9 \mathrm{eV}$ in the solid state which must be considered is the mechanism by which the dissociation actually occurs. Absorption in this region is, according to the work of Gürtler and co-workers, ${ }^{7}$ occuring into the bound $1^{1} \Sigma_{u}^{+}$ion-pair state, but as will be discussed here, considerable evidence exists to indicate that an additional absorption to an $\mathrm{Ar}^{+}\left(\mathrm{Cl}_{2}\right)^{-}$charge transfer state also occurs in this region. The obvious candidates then for the dissociation mechanism are (a) a curve crossing to a repulsive potential followed by an impulsive dissociation mechanism or (b) a harpooning mechanism mediated by the formation of the $\mathrm{Ar}^{+} \mathrm{Cl}_{2}^{-}$ charge transfer species. In the chlorine/argon system enough evidence exists to indicate that the dissociation observed at $9 \mathrm{eV}$ occurs by an impulsive mechanism. However, since the spectroscopy of $\mathrm{Cl}_{2}$ in $\mathrm{Ar}$ is quite complex in this spectral region, a detailed discussion of its features is necessary to justify the previous ascertion.

Absorption into the ion-pair $1^{1} \Sigma_{u}^{+}$state of molecular chlorine has been observed in Ne matrices, unshifted at the low energy onset with respect to the gas phase. This band was detected in Ne by Gürtler and co-workers ${ }^{7}$ by monitoring the $D^{\prime 3} \Pi_{2 g}-A^{\prime 3} \Pi_{2 u}$ "laser" emission of molecular chlorine and from the agreement at low energy with the gas phase $1^{1} \Sigma_{g}^{+} \leftarrow X^{1} \Sigma_{g}^{+}$transition, was accordingly assigned. Support for this proposal is given in Fig. 11 where we show a comparison of the Franck-Condon simulations of the $1^{1} \Sigma_{u}^{+}-X^{1} \Sigma_{g}^{+}$transition with the experimentally observed $A-X$ excitation profile. The simulation was obtained following the procedure outlined in Appendix B and using the constants pertaining to the ion-pair diabatic $1^{1} \Sigma_{u}^{+}$state potential of $\mathrm{Cl}_{2}$ as derived by Zimmerer and co-workers ${ }^{18}$ from gas phase spectroscopic data. By simply lowering the potential (i.e., decreasing the $T_{e}$ value by $0.1 \mathrm{eV}$ ) for the matrix transition we have, as shown in Fig. 11, obtained a satisfactory fit for the onset of the band, i.e., the region centered between 8.5 and $10.0 \mathrm{eV}$ (see Fig. 11). This supports Gürtler's proposal that in the matrix, the Franck-Condon accessible region of the $1^{1} \Sigma_{u}^{+}$state is the valence (ion-pair) portion, but as is clearly evident in Fig. 11, does not account for the dominant part of the $A-X$ excitation profile. This shortcoming is particularly evident at higher energies, i.e., the right hand side of Fig. 11, where the $1^{1} \Sigma_{u}^{+}$state shows no significant transition probability.

In an attempt to ascertain what transition is giving rise to absorption in this region we have carried out a FranckCondon simulation of the $2{ }^{1} \Sigma_{u}^{+}-X^{1} \Sigma_{g}^{+}$transition. This was done using the constants given in Appendix $\mathbf{B}$ by Wörmer ${ }^{19}$ for the $2{ }^{1} \Sigma_{u}^{+}$state. No significant Franck-Condon factors are obtained however for this transition in the region below $14 \mathrm{eV}$. This may be an indication that the Ryd- 
berg part of the gas phase adiabatic potential is responsible for the oscillator strength but more likely indicates that absorption to a charge transfer $\mathrm{Ar}^{+}\left(\mathrm{Cl}_{2}\right)^{-}$state occurs in this region. Simulation of a transition from the ground state of $\mathrm{Cl}_{2}$ to an $\mathrm{Ar}^{+}\left(\mathrm{Cl}_{2}\right)^{-}$state is shown in Fig. 11 and outlined in Appendix B. Thus, Fig. 11 shows that while transitions to the $1^{1} \Sigma_{\mu}^{+}$and charge transfer states account for most of the $A-X$ excitation profile, the fit is by no means complete.

The most likely source of discrepency between experiment and theory is that the $A-X$ emission can be produced from several relaxation processes and not limited to the two used in the fit shown in Fig. 11. For example, it is obvious in the experimental excitation spectrum in Fig. 11 that a maximum occuring at $10.0 \mathrm{eV}$ is clearly is not accounted for by the two state simulation. This peak at $10.0 \mathrm{eV}$ corresponds, as shown in Fig. 8, in shape and position to the large dip evident in the $\mathrm{ArCl}_{2}$ excitation spectrum and as a result, indicates the additional population of the $A$ state derived from the dissociative channel. In spite of this, a considerable region exists between the two simulated maxima where no oscillator strength is predicted. A possible explanation of this behavior would be that the electronic architecture must be considerably modified in Ar from the gas phase. This proposal can be qualitatively rationalized since the Rydberg part of the potential, present in the adiabatic gas phase potential and in $a b$ initio calculations, ${ }^{20}$ is expected to be blueshifted $^{21}$ in the matrix thus producing oscillator strength above the $1^{1} \Sigma_{u}^{+}$ion-pair region which is predicted, ${ }^{22}$ and has been shown in this study to red-shift.

A spectroscopic feature of the $\mathrm{Cl}_{2} / \mathrm{Ar}$ system which is vital in the determination of the dissociation mechanism is the excitation spectrum of $\mathrm{Ar}_{2} \mathrm{Cl}$ shown in Fig. 8(c). Thus despite the fact that the charge transfer states of $\mathrm{Ar}^{+} \mathrm{Cl}_{2}^{-}$ are calculated ${ }^{23}$ (see Fig. 11 and the extreme left of Fig. 10) to occur at energies similar to the region of the observed threshold $(9 \mathrm{eV})$, the lack of detection in excitation spectra of any regions producing atomic chlorine-related emission (i.e., $\mathrm{Ar}_{2} \mathrm{Cl}$ ), other than in the region of atomic resonances or in the $D-X, B-X$ regions of $\mathrm{ArCl}$ (see Fig. 8), rules out the possibility of a harpooning mechanism. This conclusion is justified by the following considerations. Thus, if dissociation was occuring by an $\mathrm{Ar}^{+} \mathrm{Cl}_{2}^{-}$charge transfer intermediate, then the products $\mathrm{Ar}^{+} \mathrm{Cl}^{-}$and $\mathrm{Cl}$ must result. Since the diatomic ion-pair species $\mathrm{Ar}^{+} \mathrm{Cl}^{-}$is an excited state of $\mathrm{ArCl}$ emission, which in solid $\mathrm{Ar}$ occurs as $\mathrm{Ar}_{2} \mathrm{Cl}$, would be expected at $260 \mathrm{~nm}$. Experimentally however, such a feature in the excitation spectrum of the $\mathrm{Ar}_{2} \mathrm{Cl}$ emission (more aptly described as chemiluminescence) has not, as can be seen in Fig. 8 (c), been observed. This is in contrast to the dissociation of $\mathrm{Cl}_{2}$ in xenon, ${ }^{24}$ where a chemiluminescence excitation spectrum was recorded in the region of efficient dissociation.

From the foregoing considerations it is concluded that dissociation is occuring by an impulsive mechanism following curve crossing from the $1^{\prime} \Sigma_{u}^{+}$or $\mathrm{Ar}^{+}\left(\mathrm{Cl}_{2}\right)^{-}$states to a repulsive potential correlating with ground state atomic chlorine. Identification of which absorbing state is responsible for the dissociation would, from Fig. 11, seem at hand. Thus it is obvious from Fig. 6, that the onset of efficient



FIG. 10. Potential energy surfaces accessible following excitation (upwardfacing wavy arrow) to the bound $1^{\prime} \Sigma_{u}^{+}$state of $\mathrm{Cl}_{2}$. Subsequent dissociation involving curve crossing is envisioned occuring to the repulsive potentials leading to ground state atomic chlorine asymptotes and is depicted along with other competing relaxation pathways as shown by the solid arrows. $\mathrm{Cl}_{2}$ curves and $\mathrm{Ar}^{+} \mathrm{Cl}_{2}^{-}$(dashed region) taken from Ref. 18 are supplemented by the lowest and highest energy repulsive curves (i.e., ${ }^{3} \Pi_{\mathrm{g}}$ and $2{ }^{3} \Sigma_{u}^{+}$states, respectively) Ref. 20. Relaxation routes to $\mathrm{ArCl}_{2}(360 \mathrm{~nm}$ ) and $\mathrm{Ar}_{2} \mathrm{Cl}(260 \mathrm{~nm})$ emission represented by downward-facing wavy arrows are shown schematically. Both emissions terminate on repulsive surfaces but this has not been included in the figure to avoid complications due to different coordinates. The dashed spectra shown on the left- and righthand side of the vertical energy axis represent the $\mathrm{ArCl}_{2}$ and $\mathrm{Ar}_{2} \mathrm{Cl}$ excitation spectra.

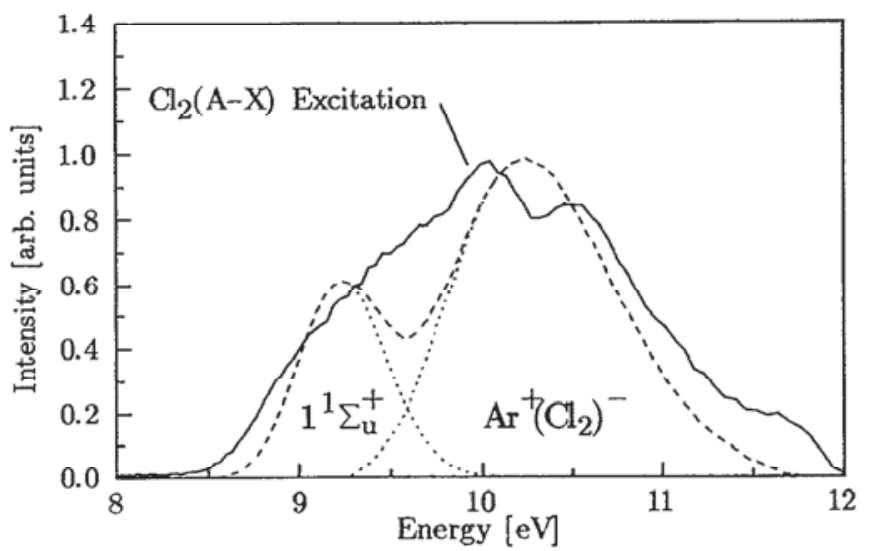

FIG. 11. Simulation of the $\mathrm{Cl}_{2} A-X$ excitation spectrum as monitored for the $v^{\prime}=0 \rightarrow v^{\prime \prime}=8$ transition at $\lambda_{\mathrm{em}}=867 \mathrm{~nm}$. The excitation spectrum is, as indicated, shown by the solid trace while the simulated curve is shown by the dashed line. Constituent components in the simulated curve are labeled and shown by the dotted lines. Transitions leading to the $1{ }^{1} \Sigma_{u}^{+}$valence state of $\mathrm{Cl}_{2}$ and the $\mathrm{Ar}^{+}\left(\mathrm{Cl}_{2}\right)^{-}$charge transfer state were obtained using the procedure outlined in Appendix B. The relative intensities of the two transitions were dictated by the fitting procedure and should not be taken to represent their relative absorption cross sections. The apparent presence of an "unaccounted for" maxima in the $A-X$ spectrum at $10.0 \mathrm{eV}$ results from extensive population of the $A$ state of $\mathrm{Cl}_{2}$ from the recombination of $\mathrm{Cl}$ atoms produced by the efficient photodissociation onset at $9.3 \mathrm{eV}$. The latter feature is resposible for the strong depletion in the $\mathrm{Ar}_{2} \mathrm{Cl}$ excitation spectrum shown in Fig. 6. 
dissociation follows the simulated absorption profile of the $\mathrm{Ar}^{+}\left(\mathrm{Cl}_{2}\right)^{-}$state closely and occurs in a region where the intensity of the simulated $1^{1} \Sigma_{u}^{+}$state is decreasing rapidly. However, as mentioned in the previous paragraph, the $\mathrm{Ar}_{2} \mathrm{Cl}$ excitation spectrum does not show this behavior. From this it can be concluded that dissociation is mediated simply by absorption to the $\mathrm{Ar}^{+}\left(\mathrm{Cl}_{2}\right)^{-}$charge transfer state and not from a harpooning mechanism. The identity of the repulsive potential involved in the impulsive dissociation mechanism is not possible but from the electronic structure calculations of Peyerimhoff and co-workers ${ }^{20}$ several canditates exist in this energy regime. Once on the repulsive potential the entire kinetic energy can be utilized in an impulsive cage escape.

A feature of the $\mathrm{Cl}_{2}$ /Ar system that is somewhat more difficult to rationalize is the relationship between the dissociation threshold and the $A-X$ excitation profile of molecular chlorine in the region of the $1^{1} \Sigma_{u}^{+}$state. From the solid line in Fig. 6, it can be seen, that the onset of the $A-X$ excitation feature follows the $\mathrm{ArCl}_{2}$ band both of which occur at much lower energies than the observed dissociation threshold. In previous work by Bondybey et al. ${ }^{1}$ it has been concluded that the $A-X$ system is populated by the recombination of atomic chlorine following absorption at $320 \mathrm{~nm}$ into the $C^{1} \Pi_{u}$ state and dissociation along this purely repulsive potential. Thus if the mechanism of the $A$ state population in the $9 \mathrm{eV}$ region occurs along the repulsive potentials correlating with ground state atomic chlorine, then an additional mechanism must be invoked to facilitate a curve crossing from the bound ion-pair $1^{\prime} \Sigma_{u}^{+}$state at energies much lower than that where permanent dissociation is observed.

It would be rather appealing to relate the curve hopping leading to permanent dissociation and that leading to $A$ state population to the same process and so allow the sharp dissociation onset at $9.3 \mathrm{eV}$ to be identified as the matrix-induced threshold. Examination of the $A-X$ excitation profile in the region of efficient permanent dissociation (around $9 \mathrm{eV}$ in Fig. 6) suggests however, that the two processes are however not related. That is, if permanent dissociation were occuring along the same repulsive potential as that leading to population of the $A$ state, the large dissociation quantum yield (0.3) measured beyond the onset should cause a depletion in the latter as has been observed for the $\mathrm{ArCl}_{2}$ excitation band. On the contrary, a peak is, as shown by the solid line in Fig. 6, present at this energy in the $A-X$ excitation profile which leads us to suggest that the surface hopping mechanism viz., the repulsive potential involved in the dissociation must be different from that populating the $A$ state.

Given that vibrational relaxation is very efficient for molecules isolated in matrices, we postulate that the $A-X$ system is accessed from vibrationally relaxed levels in the $1^{1} \Sigma_{u}^{+}$state, at which point a branching exists, leading to $\mathrm{ArCl}_{2}$ emission via a $\mathrm{Ar}^{+} \mathrm{Cl}-\mathrm{Cl}^{-}$intermediate ${ }^{7}$ or to the $A-$ $X$ emission via a repulsive potential. In the onset region of permanent dissociation, $1 \mathrm{eV}$ higher in energy at the onset of the $\mathrm{Ar}^{+}\left(\mathrm{Cl}_{2}\right)^{-}$charge transfer state, hopping to a different repulsive potential must be accessed. An attempt was made using the presently available experimental data to quantitatively determine branching ratios for the aforementioned re- laxation pathways in this energy region by comparing the relative emission intensites with absorption data. Due to the intensity of optical scattering of the samples in this energy region, inconclusive relationships have up to now been obtained.

\section{CONCLUSIONS}

The onset of $\mathrm{Cl}_{2}$ dissociation in solid argon occurs at two wavelengths, the onset at $180 \mathrm{~nm}$ corresponds to absorption into the purely repulsive ${ }^{3} \Sigma_{u}^{+}$state, the other at $130 \mathrm{~nm}$ into the bound $1^{1} \Sigma_{u}^{+}$state. As reported in our earlier paper, the quantum yield $\Phi$ for dissociation at $180 \mathrm{~nm}$ is on the order of 0.02 and the photochemical activity in this band originates exclusively from monomeric chlorine. The quantum yield for dissociation at $130 \mathrm{~nm}$ is 0.30 and independent of sample crystallinity. From spectroscopic evidence and dynamics arguments it has been concluded that cage escape occurs by a direct ballistic mechanism and not a harpooning mechanism involving a transfer of charge in the excited state. The mechanism of dissociation is envisioned as involving a curve crossing from the bound $1^{1} \Sigma_{u}^{+}$state, or more likely from the $\mathrm{Ar}^{+}\left(\mathrm{Cl}_{2}\right)^{-}$charge transfer state, to a repulsive potential on which a chlorine atom acquires sufficient kinetic energy to overcome the matrix-induced barrier. The curve-crossing mechanism is consistent with the lack of dependence of the dissociation effiency on the photon energy once above the onset of dissociation. It also explains the failure of the analytic expressions to describe the threshold curves. This work has shown that molecular chlorine clusters can be identified clearly in steady-state but much more so in time-resolved emission spectra of the $A-X$ system of $\mathrm{Cl}_{2}$. Furthermore, it has been ascertained, that in the energy region examined in the present study, cluster photochemistry plays an insignificant role in the dissociation of molecular chlorine in argon matrices.

After completion of the manuscript we were informed of preliminary results of molecular dynamics calculations carried out by Alimi and Gerber on the dissociation of $\mathrm{Cl}_{2}$ in solid Ar. The calculations which are still in progress and which will be published in the near future, ${ }^{25}$ confirm several of the proposals made in this paper. Two of these points will be mentioned here. First, calculations show that molecular chlorine does occupy a divacancy site in solid argon on deposition and that chlorine atoms occupy single substitutional sites separated by a single argon atom after dissociation. Second, the calculations reproduce the threshold at $6 \mathrm{eV}$ having a low quantum yield for dissociation and also the second threshold at $9 \mathrm{eV}$ leading to high dissociation efficiency.

\section{APPENDIX A}

The generation of the ground state potentials for $\mathrm{ArCl}$, shown in Fig. 9, followed the general procedure presented by Aquilanti and co-workers ${ }^{17}$ for the rare gas halides. The necessary potential constants are those made available to us by Aquilanti and co-workers as a private communication ${ }^{13}$ and are identical to the published values except that an additional exponential repulsive function at short internuclear separation $\left(0 \leqslant x \leqslant x_{1}\right)$ is included in the isotropic interaction 
term. Three states [Hund's case (c)] related to the "weak field" $\left|j m_{j}\right\rangle$ states of atomic $\mathrm{Cl}\left({ }^{2} P_{j}\right)$ are given analytically by

$$
\begin{aligned}
& V_{3 / 2,1 / 2}=V_{0}+\frac{1}{10} V_{2}+\frac{1}{2} \Delta-\frac{1}{2}\left(\frac{9}{25} V_{2}^{2}+\Delta^{2}-\frac{2}{3} V_{2} \Delta\right)^{1 / 2}, \\
& V_{3 / 2,3 / 2}=V_{0}-\frac{1}{3} V_{2}, \\
& V_{1 / 2,1 / 2}=V_{0}+\frac{1}{10} V_{2}+\frac{1}{2} \Delta+\frac{1}{2}\left(\frac{9}{25} V_{2}^{2}+\Delta^{2}-\frac{2}{3} V_{2} \Delta\right)^{1 / 2},
\end{aligned}
$$

where $\Delta$ is the spin-orbit splitting of atomic chlorine (109.4 $\mathrm{meV}$ ) and $V_{2}$ is the anisotropic component in the potential given by a Buckingham function

$$
V_{2}(R)=-A_{2} \exp \left(-\alpha_{2} R\right)+C_{2} / R^{6} .
$$

The following values of the constants $A_{2}=1248400.0$ $\mathrm{meV} ; \alpha_{2}=3.00 ; C_{2}=9400.0 \mathrm{meV} \AA^{6}$ have been used. $V_{0}$, the spherical contribution to the potential is described by an Exponential-spline-Morse-spline-van der Waals (ESMSW) function $f(x)$. This is scaled for the value of the internuclear separation $R m(3.88 \AA)$ at the maximum well depth $\epsilon(12.846 \mathrm{meV})$ as follows:

$$
x=R / R m \text { and } f(x)=V_{0}(R) / \epsilon .
$$

The functions, ranges, and the forms of the splines used are the following:

Exponential:

$$
f(x)=A \exp [-\alpha(x-1)], \quad 0 \leqslant x \leqslant x_{1},
$$
spline:

$$
\begin{aligned}
f(x)= & \exp \left\{a_{1}+\left(x-x_{1}\right)\left[a_{2}+\left(x-x_{2}\right)\right.\right. \\
& \left.\left.\times\left[a_{3}+\left(x-x_{1}\right) a_{4}\right]\right]\right\} \quad x_{1}<x<x_{2},
\end{aligned}
$$

Morse:

$$
\begin{aligned}
f(x)= & \exp [-2 \beta(x-1)]-2 \exp [-\beta(x-1)], \\
& x_{2} \leqslant x \leqslant x_{3},
\end{aligned}
$$

spline:

$$
\begin{aligned}
f(x)= & b_{1}+\left(x-x_{3}\right)\left\{b_{2}+\left(x-x_{4}\right)\right. \\
& \left.\times\left[b_{3}+\left(x-x_{3}\right) b_{4}\right]\right\}, \quad x_{3}<x<x_{4},
\end{aligned}
$$

van der Waals:

$$
f(x)=-\left(C_{0} / \epsilon R m^{6}\right) x^{-6}, \quad x_{4} \leqslant x .
$$

Constants used in the above equations had the following values:

$\beta=6.2, \quad C_{0}=51648.0 \mathrm{meV} \AA^{6}$,

$A=0.350, \quad \alpha=15.00$,

$x_{1} . . x_{4}=0.69,0.81,1.15,1.50$ (scaled spline positions),

$a_{1} . . a_{4}=3.6002, \quad-18.3395,-27.8290, \quad-45.6908$

(spline constants, exponential-Morse),

$b_{1} . . b_{4}=-0.6334, \quad 1.5142, \quad-4.1368,2.8363$

(spline constants, Morse-van der Waals).

\section{APPENDIX B}

Franck-Condon simulation of the $1^{1} \Sigma_{u}^{+}-X^{1} \Sigma_{g}^{+}$absorption, shown in Fig. 11, was undertaken with a computer program described already in a previous paper. ${ }^{6}$ The constants used in generating the excited state potentials were taken from the data of Wörmer et al.. ${ }^{18}$ To obtain the excited state potentials in the matrix, the approach used involved the extraction of the diabatic potentials corresponding to the valence parts of the adiabatic $1^{1} \Sigma_{u}^{+}$and $2{ }^{1} \Sigma_{u}^{+}$gas phase curves. The potential $V_{1}$ of the $1^{1} \Sigma_{u}^{+}$state, as a function of the internuclear separation $r$, was given by a Rittner potential, modified by an attractive Morse component, as follows:

$$
\begin{aligned}
V_{1}(r)= & -(Z / r)+E_{1}+B_{1} e^{-\beta_{1} r} \\
& +A\left[\left(1-e^{-\alpha\left(r-r_{e}\right)}\right)^{2}-1\right] .
\end{aligned}
$$

The constants pertaining to the Rittner function are the following: $Z=116645 \mathrm{~cm}^{-1}, \quad E_{1}=108104 \mathrm{~cm}^{-1}$, $B_{1}=959069 \mathrm{~cm}^{-1}, \beta_{1}=1.84917 \AA^{-1}$ and to the Morse function; $A=6500 \mathrm{~cm}^{-1}, \alpha=1.04 \AA^{-1}$, and $r_{e}=2.7 \AA$. For the $2{ }^{1} \Sigma_{u}^{+}$state a simple Rittner function

$$
V_{2}(r)=-(\dot{Z} / r)+E_{2}+B_{2} e^{-\beta_{2} r},
$$

given in Ref. 19 by Wörmer, was used with the following constants: $E_{2}=123904 \mathrm{~cm}^{-1}, B_{2}=1.3 \times 10^{9} \mathrm{~cm}^{-1}$ and $\beta_{2}=4.74 \AA^{-1}$. It should be mentioned that although the 1 and $2{ }^{1} \Sigma_{u}^{+}$states appear at first glance to be rather similar, i.e., both are ion-pair states correlating to different asymptotes of $\mathrm{Cl}^{-}$, the inclusion of the attractive Morse function in the $1^{\prime} \Sigma_{u}^{+}$potential lowers the Franck-Condon accessible region considerably, so that it ends up in the $8-10 \mathrm{eV}$ region. Conversely, the absence of this feature in the $2^{\prime} \Sigma_{u}^{+}$state explains why as mentioned in the text, the gas phase potential data by Wörmer ${ }^{19}$ does not explain absorption in the region between 10 and $12 \mathrm{eV}$. For the ground state of $\mathrm{Cl}_{2}$ a Hubert-Hirschfelder potential using the constants given by Herzberg ${ }^{26}$ was used.

The approach used to simulate the transitions of ground state $\mathrm{Cl}_{2}$ to an $\mathrm{Ar}^{+}\left(\mathrm{Cl}_{2}\right)$ charge transfer state is that outlined by Fajardo et al., ${ }^{27}$ using the data of Chen and Wentworth. ${ }^{28}$ The transition energy $E_{C T}$ to such a charge transfer state can be estimated from the following equation: ${ }^{27}$

$$
E_{C T}=\mathrm{IP}(\mathrm{Ar})-\mathrm{EA}\left(\mathrm{Cl}_{2}\right)+\Delta V\left(r_{c}\right)
$$

with $\operatorname{IP}(\mathrm{Ar})$ the ionization potential of $\mathrm{Ar}, \mathrm{EA}\left(\mathrm{Cl}_{2}\right)$ the electron affinity of $\mathrm{Cl}_{2}$ and the term $\Delta V\left(r_{\mathrm{c}}\right)$ which combines the binding and polarisation energies of $\mathrm{Ar}^{+}-\mathrm{Cl}_{2}^{-}$species. The width of the absorption band is determined in this model by the variation of the "vertical" electron affinity of $\mathrm{Cl}_{2}$ with the $\mathrm{Cl}-\mathrm{Cl}$ separation in the vibrationless level of the electronic ground state. ${ }^{28}$ In other words, the shape is determined by the square of the ground state vibrational wave function reflected on the $\mathrm{Cl}_{2}^{-}$potential. The former was determined by numerically solving the radial Schrödinger equation ${ }^{6}$ while for the latter, a delta function was used to represent the continuum wave function of the repulsive $\mathrm{Cl}_{2}^{-}$ potential, in accordance with the Condon approximation. The shape predicted using the equation given above is represented by the dotted/dashed line shown and labeled on the right in Fig. 11.

\section{ACKNOWLEDGMENTS}

This work was financed by the Bundesminesterium für Forschung und Technologie (BMFT) via Contract No. 
413AXI, TP5 and the Deutsche Forschungsgemeinschaft via the Sonderforschungsbereich 337. J.McC. is grateful to the Alexander von Humboldt Foundation for financial support. Gerald Zerza and Xu Jiang are acknowledged for their assistance in measuring laser induced $\mathrm{Cl}_{2} \mathrm{~A}-\mathrm{X}$ emission in thin films and crystals, respectively. We are grateful to Dr. Cappelletti in the Acquilanti group for making the data necessary for the generation of the $\mathrm{ArCl}$ potentials available to us prior to publication.

${ }^{1}$ V. E. Bondybey and C. Fletcher, J. Chem. Phys. 64, 3615 (1976).

${ }^{2}$ J. Langen, K.-P. Lodemann, and U. Schurath, Chem. Phys. 112, 393 (1987); J. Langen, dissertation, University of Bonn, 1985 (unpublished).

${ }^{3}$ P. Beeken, M. Mandich, and G. Flynn, J. Chem. Phys. 76, 5995 (1982); M. Mandich, P. Beeken, and G. Flynn, ibid. 77, 702 (1982).

${ }^{4}$ M. E. Fajardo and V. A. Apkarian, J. Chem. Phys. 89, 4124 (1988).

${ }^{5}$ R. Alimi, A. Brokman, and R. B. Gerber, in Stochasticity and Intramolecular Redistribution of Energy, edited by R. Lefebvre and S. Mukamel (Reidel, Dodrecht, 1987); J. Chem. Phys. 91, 1611 (1989).

${ }^{6}$ H. Kunz, J. G. McCaffrey, R. Schriever, and N. Schwentner, J. Chem. Phys. 94, 1039 (1991). Please note that the pictures appearing as 2, 3, and 5 have been interchanged and belong to the captions numbered Fig. 5, Fig. 2 , and Fig. 3, respectively.

${ }^{7}$ P. Gürtler, H. Kunz, and J. LeCalvé, J. Chem. Phys. 91, 6020 (1989).

${ }^{8}$ T. Kessler, R. Markus, H. Nahme, and N. Schwentner, Phys. Status Solidi B 139, 619 (1987)

${ }^{9}$ B. S. Ault, W. F. Howard, and L. Andrews, J. Mol. Spectrosc. 55, 217 (1975).

${ }^{10}$ M. E. Fajardo, R. Whitnall, J. Feld, F. Okada, W. Lawrence, L. Wiedeman, and V. A. Apkarian, Laser Chem. 9, 1 (1988).

" R. Schriever, M. Chergui, Ö. Ünal, N. Schwentner, and V. Stepanenko, J. Chem. Phys. 93, 3245 (1990).
${ }^{12}$ C. E. Moore, Atomic Energy Levels, Natl. Bur. Stand., Circ. No. 467 (U.S. GPO, Washington, D.C., 1957), Vol. 1, p. 195.

${ }^{13} \mathrm{D}$. Cappelletti (private communication, 1990).

${ }^{14}$ C. K. Kittel, Introduction to Solid State Physics, 5th ed. (Wiley, New York, 1976).

${ }^{15}$ B. P. Reid, K. C. Janda, and N. Halberstadt, J. Phys. Chem. 92, 587 (1988); D. D. Edvard, J. I. Cline, and K. C. Janda, J. Chem. Phys. 88, 5433 (1988).

${ }^{16}$ K.-H. Gödderz, J. G. McCaffrey, and N. Schwentner (manuscript in preparation).

${ }^{17}$ V. Acquilanti, E. Luzzatti, F. Pirani, and G.-G. Volpi, J. Chem. Phys. 98, 6165 (1988); V. Acquilanti, D. Cappelletti, E. Luzzatti, and F. Pirani, Chem. Phys. 145, 293 (1990).

${ }^{18} \mathrm{~J}$. Wörmer, T. Möller, J. Stapelfeld, G. Zimmerer, D. Haaks, and S. Kampf, J. LeCalvé, and M.-C. Castex, Z. Phys. D 7, 383 (1988).

19 J. Wörmer, Internal Report DESY/HASYLAB, 87-03, March 1987.

${ }^{20}$ S. D. Peyerimhoff and R. J. Buenker, Chem. Phys. 57, 279 (1981); F. Grein, S. D. Peyerimhoff, and R. J. Buenker, Can. J. Phys. 62, 1928 (1984).

${ }^{21}$ M. Chergui, N. Schwentner, and W. Böhmer, J. Chem. Phys. 85, 2472 (1986).

${ }^{22}$ H. Jara, H. Pummer, H. Egger, M. Shadidi, and C. K. Rhodes, Phys. Rev. B 34, 7534 (1986).

${ }^{23}$ T. Möller, B. Jordon, G. Zimmerer, D. Haaks, J. LeCalvé, and M.-C. Castex, Z. Phys. D 4, 73 (1986).

${ }^{24}$ J. G. McCaffrey, H. Kunz, and N. Schwentner, J. Chem. Phys. (submitted).

${ }^{25}$ R. Alimi, B. Gerber, H. Kunz, J. G. McCaffrey, and N. Schwentner (manuscript in preparation).

${ }^{26} \mathrm{~K}$. P. Huber and G. Herzberg, Molecular Spectra and Molecular Structure, IV, Constants of Diatomic Molecules (Van Nostrand Rheinhold, New York, 1979).

${ }^{27}$ M. E. Fajardo, V. A. Apkarian, A. Moustakas, H. Kreuger, and E. Weitz, J. Phys. Chem. 92, 357 (1988).

${ }^{28}$ E. C. M. Chen and W. E. Wentworth, J. Phys. Chem. 89, 4099 (1985). 
The Journal of Chemical Physics is copyrighted by the American Institute of Physics (AIP). Redistribution of journal material is subject to the AIP online journal license and/or AIP copyright. For more information, see http://ojps.aip.org/jcpo/jcpcr/jsp Copyright of Journal of Chemical Physics is the property of American Institute of Physics and its content may not be copied or emailed to multiple sites or posted to a listserv without the copyright holder's express written permission. However, users may print, download, or email articles for individual use. 
The Journal of Chemical Physics is copyrighted by the American Institute of Physics (AIP). Redistribution of journal material is subject to the AIP online journal license and/or AIP copyright. For more information, see http://ojps.aip.org/jcpo/jcpcr/jsp 